\title{
Aplicación individual del programa de entrenamiento metacognitivo en la esquizofrenia: dos estudios de caso
}

\author{
CRISTINA MONFORT ESCRIG \\ al073891@uji.es \\ Josep PENA-GARIJO \\ jpena@uji.es
}

\section{Resumen}

Introducción: El programa de entrenamiento metacognitivo (MCT) es una intervención dirigida a los sesgos, creencias y errores cognitivos en la esquizofrenia. Con el objetivo de evaluar la eficacia de esta intervención en la sintomatología de dos sujetos, se hipotetizó que el MCT: 1) disminuirá los síntomas psicóticos positivos y negativos 2) fomentará interpretaciones y atribuciones apropiadas y 3) mejorará la calidad de vida, conciencia de enfermedad y estado de ánimo. Método: Diseño de caso único subtipo $A B$ (pre y post evaluación) y posterior seguimiento. Se utilizaron los siguientes instrumentos: Escala de síntomas positivo y negativo (PANSS), Inventario de ideas delirantes de Peters-21 (PDI-21), Inventario de depresión de Beck II (BDI-II), Escala de la Calidad de Vida (QLS) y Escala de la discapacidad (WHO/DAS). La intervención fue aplicada a dos varones diagnosticados de: a) Esquizofrenia de tipo indiferenciado y b) esquizofrenia paranoide. Resultados: Se observaron cambios clínicamente significativos en la psicopatología y el funcionamiento psicosocial de ambos sujetos. Se observó una reducción más significativa para los síntomas positivos e ideas delirantes. La evaluación psicosocial destacó por una mejoría de la conciencia de enfermedad, funciones intrapsíquicas e interpersonales y funcionamiento familiar. Conclusiones: El MCT fue eficaz en ambos casos, mejorando los síntomas positivos (ideas delirantes), el insight y la conciencia de enfermedad, y la calidad de vida y el funcionamiento psicosocial general de ambos sujetos.

Palabras clave: metacognitivo, МСт, esquizofrenia, caso único.

\footnotetext{
Abstract

Introduction: The Metacognitive Training for Schizophrenia (MCT) is an intervention that addresses biases, beliefs and cognitive errors. In order to evaluate the efficacy of this intervention in the psychopathology of two subjects, we hypothesized that МСT: 1) decreases the positive and negative symptoms 2) promotes appropriate interpretations and attributions and 3) improves the quality of life, insight and mood. Method: A single case design based on subtype $A B$ (pre and post) and follow-up was evaluated by following assessment instruments: Positive and Negative Symptoms Scale (PANSS), Peters Delusions Inventory-21 (PDI-21), Beck Depression Inventory II (BDI-II), the Quality of Life Scale (QLS) and the Disability Scale (WHO/DAS). The intervention was imple-
} 
mented in two outpatient males diagnosed of A) Chronic Undifferentiated Schizophrenia and B) Paranoid Schizophrenia. Results: Clinically significant changes in psychopathology and psychosocial functioning in both subjects were observed. Regarding psychopathology, a significant reduction of positive symptoms and delusions was observed, whereas no changes were observed in the BDI-II. The psychosocial assessment highlights a significant improvement in awareness of cognitive biases, intrapsychic and interpersonal functions and family functioning. Conclusions: The present study shows the efficacy of an individualized MCT program improving positive symptoms, delusional thinking, insight and awareness of cognitive biases, as well as quality of life and psychosocial functioning in both cases.

Keywords: metacognitive, MCT, schizophrenia, single case.

\section{Introducción}

El tratamiento de elección para la esquizofrenia ha venido siendo la intervención farmacológica con antipsicóticos, los cuales que han mostrado grandes beneficios en la reducción de la sintomatología. No obstante, muchos pacientes no responden a ellos y tan solo producen mejorías limitadas en los síntomas negativos, funciones cognitivas, funcionamiento social y calidad de vida (Kane, 2007; Lemos y cols., 2009).

En este sentido, intervenciones tales como la terapia cognitivo-conductual, han demostrado eficacia en la reciente investigación. Pero también, nuevos enfoques terapéuticos como el entrenamiento metacognitivo han demostrado ser un complemento o incluso una alternativa a los tratamientos al uso.

El entrenamiento metacognitivo para la esquizofrenia (МСт; МСT+ para la versión individual) es una intervención basada en conceptos tradicionales de la psicoeducación, la rehabilitación cognitiva, el entrenamiento en cognición social y teoría de la mente (TOM) y la terapia cognitivo-conductual (TCC). El programa tiene como objetivo modificar errores y sesgos cognitivos comunes en la esquizofrenia, partiendo del supuesto de que estas distorsiones pueden evolucionar hasta la creación de falsas creencias o, en última instancia, convertirse en delirios (Freeman, 2007; Moritz y Woodward, 2007; Moritz, Vitzthum, Randjbar, Veckenstedt y Woodward, 2010). El programa se desarrolla a través de ocho módulos cuyas sesiones tienen por objeto aumentar la conciencia del paciente sobre estas distorsiones y enseñarles a reflexionar sobre ellas de una forma crítica, a complementar, a cambiar y a ampliar su actual repertorio de solución de problemas y sus habilidades cognitivas.

Una serie de estudios, en su mayoría pequeños y medianos, han investigado la aceptación y la eficacia del entrenamiento metacognitivo (Moritz y cols., 2014). Los resultados han mostrado mejorías significativas, sobre todo en combinación con los tratamientos habituales, en los síntomas psicóticos y en los sesgos cognitivos. Varios estudios han demostrado una reducción considerable en la gravedad de los delirios y una mejoría en el conocimiento de los engaños, en la atribución de los delirios y en el conocimiento de la enfermedad, acompañado también por una reducción en los síntomas depresivos (Favrod, Maire, Bardy, Pernier y Bonsack, 2011: Favrod y cols., 2013). El MCT, en comparación con los tratamientos habituales, mostró también una mejoría de los síntomas positivos (Kumar, Zia UI Haq, Dubey y cols., 2010; Moritz, Kerstan, Veckenstedt y cols., 2011). Los resultados del estudio de Naughton, Nulty, Abidin, Davoren, O'Dwyer y Kennedy (2012) demostró que los pacientes expuestos al MCT 
mejoraron su capacidad de dar consentimiento al tratamiento y que, cuantas más sesiones recibían, mayor fue la mejoría en esta capacidad de toma de decisiones, debido principalmente a la mejoría en la comprensión y el razonamiento.

Otro aspecto relevante del programa МСт es su posible influencia en los síntomas negativos de la esquizofrenia, habida cuenta de que los actuales tratamientos farmacológico y psicológico proporcionan hasta ahora un beneficio bastante modesto. Un reciente estudio (McLeod y cols., 2014) ha mostrado que la adición de las puntuaciones de metacognición mejora los resultados de predictores ya conocidos de los síntomas negativos como la escala PANSS. Estos resultados sugieren que los errores en el procesamiento cognitivo podrían ser determinantes importantes de la expresión de los síntomas negativos durante las primeras etapas de la psicosis.

La evidencia de la eficacia del MCT, aunque todavía preliminar, subraya la importancia del programa como complemento a la terapia habitual de los síntomas positivos, ya que mejora los sesgos cognitivos y el insight en la esquizofrenia (Moritz y cols., 2014). En este sentido, el presente estudio pretende observar y evaluar los cambios clínicos y psicosociales en dos sujetos diagnosticados de esquizofrenia, como paso previo a diseñar un estudio más complejo sobre la influencia de los sesgos cognitivos en la esquizofrenia y otros trastornos del espectro psicótico.

\section{Método}

\section{Objetivo}

Evaluar los efectos de la aplicación de un programa de entrenamiento metacognitivo sobre la sintomatología presente en dos sujetos con diagnósticos clínicos de esquizofrenia. Se hipotetizó que el entrenamiento metacognitivo: 1) disminuirá la presencia de psicopatología psicótica positiva y negativa; 2) fomentará la generación de interpretaciones y atribuciones de los acontecimientos más apropiados; 3) mejorará la calidad de vida general, la conciencia de enfermedad y el estado de ánimo.

\section{Diseño}

Se trata de un diseño cuasi-experimental de caso único de tipo AB con pre- y post-evaluación y posterior seguimiento. Los dos sujetos del estudio fueron expuestos a dos condiciones experimentales. En la primera (A) se llevó a cabo la evaluación del problema para establecer la línea de base, mientras que en la segunda (B) se aplicó el tratamiento correspondiente, llevándose a cabo evaluación post-intervención de las medidas objetivo y un posterior seguimiento a los 1,5 meses.

\section{Sujetos}

La intervención fue aplicada a dos pacientes procedentes de la Unidad de Salud Mental Illes Columbretes del Hospital General de Castelló.

El sujeto A era un varón de 43 años de edad, soltero, estudios medios y de nivel socioeconómico medio que cumplía criterios diagnósticos DSM-IV-TR (APA, 2004) para esquizofrenia de Tipo Indiferenciado de curso crónico con predominio de síntomas negativos. El inicio de la enfermedad se estima hace 4 años coincidiendo con un presunto episodio de acoso laboral. El 
paciente ha ido evolucionando hacia un estancamiento emocional en el suceso del despido con proporciones de ideación delirante, síntomas apático-búlicos, escaso insight y poca motivación de cambio. Estos síntomas han afectando a la convivencia familiar con descontrol de impulsos, consumo de alcohol y posteriores soliloquios psicóticos y heteroagresividad verbal.

El sujeto B era un varón de 40 años de edad, soltero, con estudios superiores, que convive en el domicilio familiar con su madre y abuela con integración a las labores doméstica y cumple criterios para esquizofrenia paranoide de curso crónico. El paciente lleva tratamiento psiquiátrico desde hace veinte años por continuos episodios psicóticos de tipo delirante que se iniciaron en un permiso del servicio militar. El curso ha evolucionado con marcadas alteraciones en la forma y contenido del lenguaje que se caracterizan por un discurso tangencial, circunstancial y con pararespuestas que repercuten en la aparición generalizada de ideación esquizotípica de los acontecimientos y experiencias vitales del sujeto.

Las pautas farmacológicas no se vieron modificadas durante la intervención en ninguno de los sujetos.

\section{Instrumentos}

1. Entrevista clínica estructurada para trastornos de eje I del DSM IV (SCID-I). Permite establecer los diagnósticos clínicos más importantes del eje I del DSM-IV (APA, 1994) de una forma totalmente estandarizada, la prevalencia del trastorno, problemas psicosociales y ambientales y, la actividad global. Su duración oscila entre 45 y 90 minutos, y ha mostrado unos índices de fiabilidad y validez adecuados.

2. Escala de síntomas positivo y negativo (PANSS). Desarrollada por Kay y colaboradores (1987) y basada en la Brief Psychiatric Rating Scale (BPRS), la PANSS consta de 30 ítems que evalúan el síndrome esquizofrénico desde una doble perspectiva: dimensional, que evalúa la gravedad del síndrome positivo, del negativo y de la psicopatología general del trastorno esquizofrénico y, categorial, que clasifica el trastorno esquizofrénico en positivo, negativo o mixto. Se evalúa mediante una entrevista semi-estructurada de 30-40 minutos de duración y consta de 30 ítems (síntomas).

3. Adaptación española del inventario de ideas delirantes de Peters-21 (PDI-21). Diseñada para la valoración de los síntomas delirantes en la población general (Peters, Joseph, Day y Garety, 2004). La adaptación española del PDI-21 (López-llundain, Pérez-Nievas, Otero y Mata, 2006) es un instrumento heteroaplicado que consta de un total de 21 ítems en formato de respuesta dicotómica sí/no. Además cuenta con tres subescalas que miden el grado de convicción, preocupación y estrés.

4. Inventario de depresión de Beck (BDI-II). Es una escala autoaplicada de 21 ítems que proporciona una medida de la presencia y de la gravedad de la depresión (Beck, Ward, Mendelson, Mock y Erbaugh, 1961). Permite establecer cortes para clasificar entre: mínima depresión, depresión leve, depresión moderada y depresión grave.

5. Escala de la calidad de vida (QLS) (Heinrichs, Hanlon y Carpenter, 1984). Desarrollada para evaluar la disfunción del paciente debido al defecto esquizofrénico. Sin embargo, en la mayor parte de los estudios se aplica con la intención de determinar el nivel de calidad de vida del paciente. La escala consta de 21 ítems que se agrupan en cuatro categorías o factores: funciones intrapsíquicas, relaciones interpersonales, rol instrumental y el uso de objetos comunes y actividades cotidianas.

6. Escala de la discapacidad de la Organización Mundial de la Salud (WHO/DAS). La DAS es un instrumento sencillo heteroaplicado desarrollado por la OMS (1988) para la valoración clínica de las limitaciones que presentan los enfermos psiquiátricos graves. 
Consta de 7 ítems que se agrupan en 3 apartados: período cubierto por la valoración, áreas específicas de funcionamiento (dimensiones) y capacidades específicas del sujeto.

\section{Procedimiento}

Los pacientes fueron derivados por un psiquiatra de la unidad. Se requirió el consentimiento informado y se les explicó la finalidad de la intervención. Ésta se llevó a cabo durante un total de 15 sesiones individuales. Las sesiones se distribuyeron según las fases definidas del estudio, así como otras consideraciones propias de la fase de evaluación y de la adecuación del tratamiento a los pacientes. Por último, se realizó una evaluación de seguimiento a los 1,5 meses de haber finalizado la intervención.

\section{Resultados}

El análisis de las diferencias entre puntuaciones puede considerarse clínicamente significativo a partir de los cortes propuestos por los instrumentos, así como de la comparación y concordancia de los resultados con otros estudios. Un análisis más concreto de la efectividad del tratamiento se ha realizado mediante el análisis visual de gráficos para cada instrumento de evaluación a nivel psicopatológico y del funcionamiento psicosocial y de la calidad de vida.

\section{Psicopatología}

Las puntuaciones obtenidas en la Escala para el síndrome positivo y negativo de la esquizofrenia (PANSS) muestran para ambos casos una disminución de los síntomas de las escalas positiva, negativa y psicopatología general. La disminución de los síntomas en el caso A, aunque es menos significativa para las tres subescalas, muestra una mejoría clínica de los síntomas negativos, así como de la psicopatología general (figura 1). En el caso B, la disminución de los síntomas psicóticos para las tres subescalas es importante, sobre todo la escala de síntomas positivos (figura 2). Las puntuaciones se mantienen estables en la primera evaluación de seguimiento.

La evaluación realizada con el Inventario de ideas delirantes de Peters-21 (PDI-21) pone de manifiesto para el caso A, ideas delirantes enmascaradas de contenido autorreferencial y religioso, que se han visto disminuidas levemente en los tres niveles de gravedad (figura 1). En el caso B, donde destacaba un buen ajuste intrapsíquico sobre la creencia de las ideas delirantes, disminuyeron los niveles de las tres subescalas (figura 2), manteniéndose estables en la fase de seguimiento.

Los resultados obtenidos mediante el Inventario de depresión de Beck (BDI-II) no mostraron cambios en las puntuaciones de ambos sujetos. Se mantuvo un grado de depresión leve para el caso A y ausencia de depresión para el caso B. 

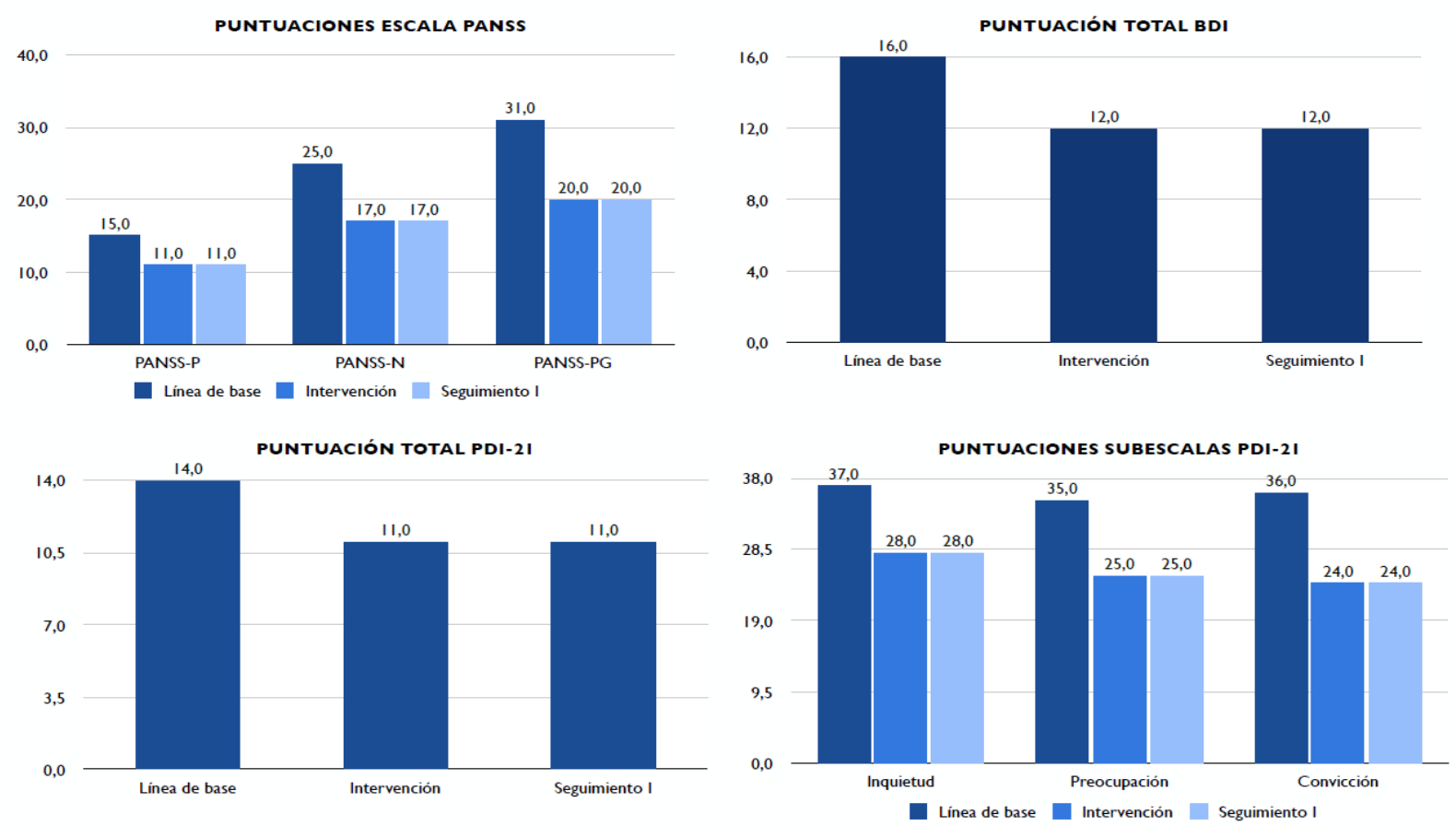

Figura 1. Caso A, escalas psicopatología
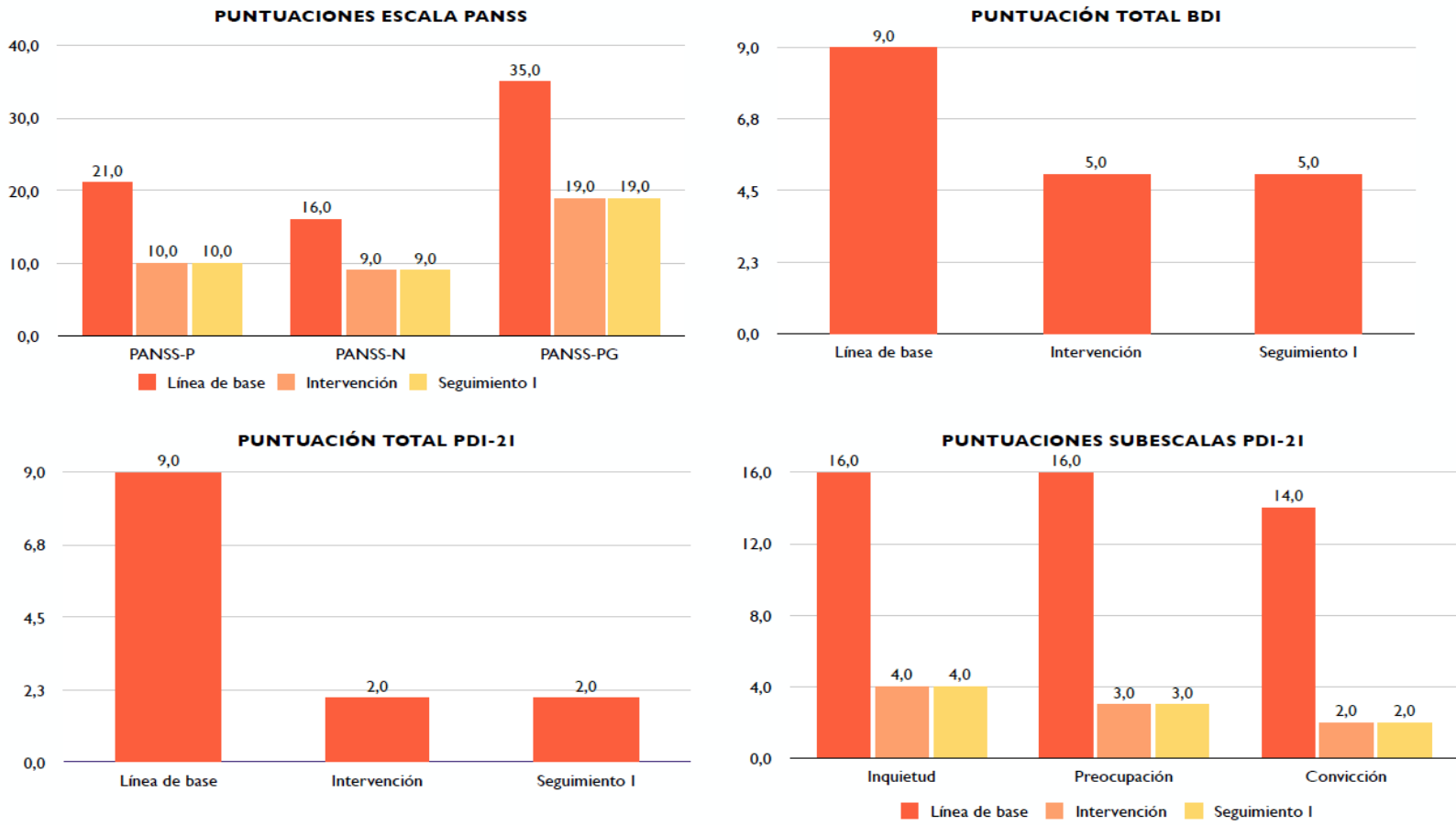

Figura 2. Caso B, escalas psicopatología 


\section{Funcionamiento psicosocial y calidad de vida}

La calidad de vida y el funcionamiento psicosocial han sido evaluados con la Escala de la calidad de vida (QLS) y la Escala de discapacidad de la Organización Mundial de la Salud (WHO/ DAS), siendo los resultados para ambos pacientes clínicamente menos significativos al cambio. Los resultados de la calidad de vida del caso A (figura 3), destacan una mayor conciencia de enfermedad y motivación de cambio, aunque el funcionamiento psicosocial sigue manteniéndose en niveles bajos. Por lo que se refiere a la evaluación de la discapacidad, denota una leve mejoría en el funcionamiento familiar y social inmediato (figura 3). Los resultados del segundo paciente (figura 4) muestran una mejoría de la calidad de vida con niveles significativos para las funciones intrapsíquica e interpersonales, con inicio de contacto social, y mayor predisposición y motivación hacia actividades de ocupación del tiempo en el ámbito familiar y social. Por otro lado, la evaluación de la discapacidad muestra un incremento de los niveles de funcionamiento ocupacional y social. Las puntuaciones se mantienen estables para la fase de seguimiento.

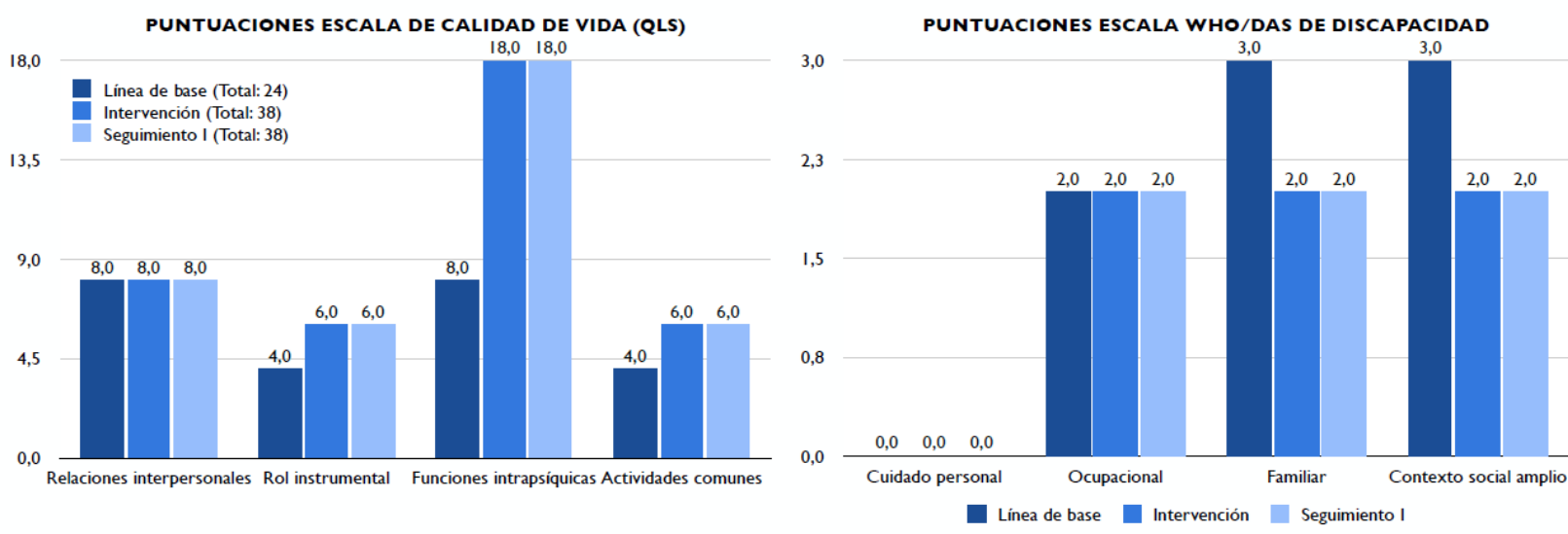

Figura 3. Caso A, escalas psicosociales

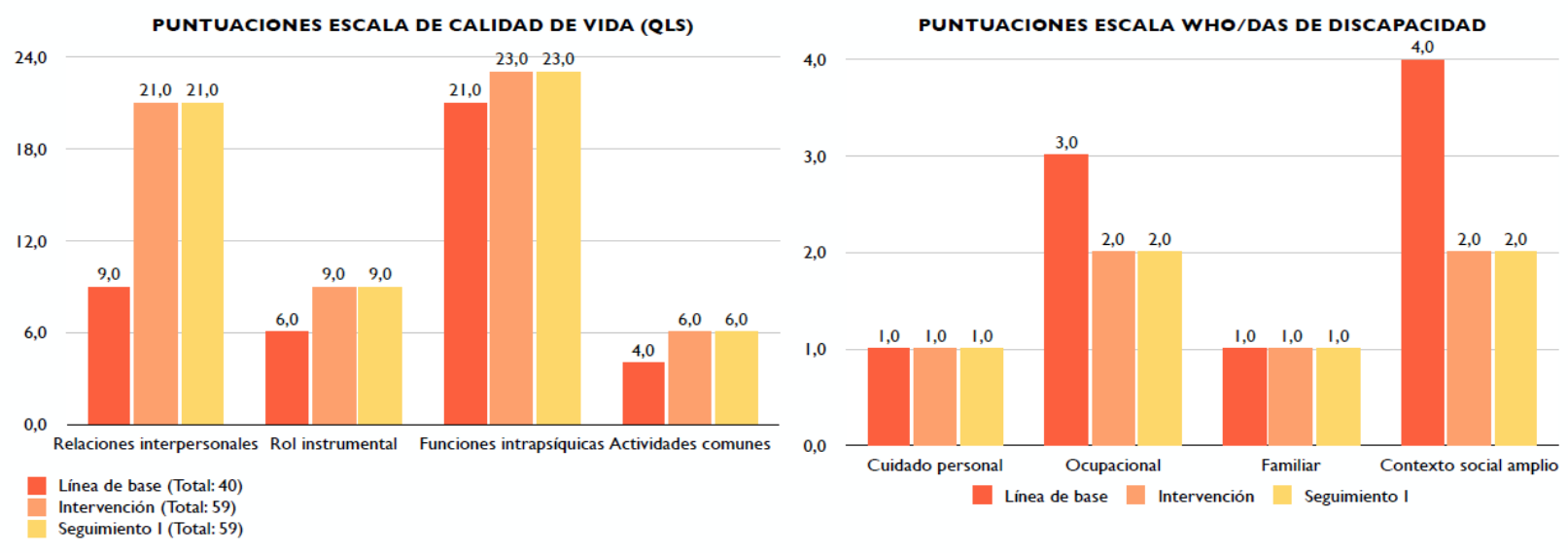

Figura 4. Caso B, escalas psicosociales 


\section{Discusión y conclusiones}

\section{Efectos del entrenamiento metacognitivo}

Los resultados indican un cambio significativo en las variables psicopatológicas y de funcionamiento psicosocial de ambos sujetos a partir de la inclusión en el tratamiento, de acuerdo a las hipótesis del estudio. Los resultados obtenidos han demostrado cambios clínicamente significativos tras la aplicación del tratamiento en los valores de la Escala para el síndrome positivo y negativo (PANSS). Ambos pacientes, que referían severas sintomatologías psicóticas, en el primero de los casos de contenido negativo y en el segundo de desorganización conceptual, han visto disminuidos tales síntomas a niveles de significación entre leves (caso A) y moderados (caso B), siendo la reducción más significativa para los síntomas positivos. Estos resultados están de acuerdo con los de otros estudios sobre la efectividad del entrenamiento metacognitivo en la disminución de la sintomatología psicótica de los pacientes con esquizofrenia. En el estudio de Favrod y cols. $(2010,2013)$ los resultados mostraron una mejoría considerable en el pensamiento delirante, confirmados también por los resultados en las escalas PSYRATS y SUMD. Por otro lado, los resultados del estudio de Kumar y cols. (2010) mostraron una reducción significativa de los síntomas positivos de la escala PANSS. Esta reducción fue también más significativa en comparación a los resultados del grupo que solo fue expuesto a tratamiento habitual (TAU). También se encontró mejoría en otro estudio sobre la efectividad del EMC individual (Moritz y cols., 2011) para los síntomas positivos, sobre todo, los delirios.

Las puntuaciones del Inventario de ideas delirantes de Peters (PDI-21) mostraron mejoría en el insight y conciencia de las ideas delirantes en ambos pacientes. Estos resultados, de acuerdo a las hipótesis planteadas, sugieren una creciente generación de interpretaciones y atribuciones más apropiadas y un aumento de la conciencia de enfermedad. Estos datos están en consonancia con los del estudio de Favrod y cols. (2010) que encontraron un progreso estadísticamente significativo en la atribución de los delirios a la enfermedad y la conciencia de enfermedad y necesidad de tratamiento, evaluados con la escala SUMD. Por otro lado, el estudio de Naughton y cols. (2012) mostró también una mejoría en la comprensión y razonamiento de la necesidad de tratamiento evaluados con la escala MacCAT-T.

Sin embargo, los resultados de la evaluación del estado de ánimo con el Inventario de Depresión de Beck (BDI) no han sido clínicamente significativos, a diferencia del estudio de Favrod y cols. (2010), en el que sí se observó una reducción de la escala de depresión de la PANSS. Esto pudo deberse a que, en nuestro caso, utilizamos el BDI, que es una medida más sensible y más relacionada con los cambios producidos en la terapia cognitiva. En la intervención que hemos implementado, los síntomas depresivos no se abordan directamente, pudiendo esto justificar por qué no se han observado cambios.

Por otro lado, cabe señalar también los cambios producidos en ambos sujetos en la calidad de vida y el funcionamiento psicosocial medidos por los valores de la Escala de calidad de vida (QLS) y la Escala de discapacidad de la OMS (WHO/DAS). En ambos casos se produjo una mejoría clínicamente significativa de leve (caso A) a moderada (caso B), tanto en las relaciones interpersonales y funciones intrapsíquicas de la calidad de vida como en el funcionamiento social. Estos resultados están a favor de la hipótesis de que el entrenamiento metacognitivo mejora la calidad de vida general y el funcionamiento social de los pacientes. En el estudio de Naughton y cols. (2012), los resultados también mostraron mejoría en el nivel de funcionamiento psicosocial de la escala GAF. Por otro lado, el estudio de Moritz y cols. (2011) mostró también 
una mejoría significativa en la percepción de la calidad de vida, sobre todo referida al bienestar psicológico y en las relaciones sociales.

\section{Limitaciones y nuevas perspectivas}

El estudio diseñado desde el enfoque cuasi-experimental con un programa de intervención bien estructurado, se ciñe a los efectos de la intervención individual, por lo que estos resultados no pueden extrapolarse a la eficacia del entrenamiento metacognitivo en general sino al efecto particular sobre los casos aquí descritos. No obstante, aunque no se han obtenido estadísticos de significación debido a la limitación de los sujetos muestrales, los resultados analizados mediante análisis visual gráfico parecen ir en consonancia con los resultados de otros estudios reseñados en el documento. A pesar de estas limitaciones, este estudio abre las puertas a futuras vías de investigación en el campo de la intervención clínica y psicosocial. El estudio aquí presentado puede tomarse como una investigación preliminar a otras más exhaustivas con una muestra de sujetos mayor, con grupos control y con diseños aleatorizados que permitan extraer inferencias sobre factores clínicos, psicosociales o de efectividad del tratamiento en trastornos del espectro psicótico o, incluso, en otros trastornos clínicos en los que se evidencian errores y sesgos cognitivos similares.

Finalmente, podemos considerar que el programa de entrenamiento metacognitivo que hemos descrito fue un tratamiento eficaz en los dos casos estudiados, sobre todo, si tenemos en cuenta que ambos pacientes estaban siendo tratados en la Unidad de Salud Mental desde hacía tiempo y que, sobre todo en uno de ellos, la mejoría había sido muy limitada y la tendencia de los síntomas era hacia la cronicidad.

\section{Referencias bibliográficas}

American Psychiatric Association (2000). Diagnostic and statistical manual of mental disorders (4th edition revised) Washington, DC: American Psychiatric Publishing.

American Psychiatric Association (2013). Diagnostic and Statistical Manual of Mental Disorders (5th edition). Arlington: VA: American Psychiatric Publishing.

Beck, A., Ward, C., Mendelson, M., Mock, J. y Erbaugh, J. (1961). An inventory for measuring depression. Archives of General Psychiatry, 4, 561-571.

Favrod, J., Maire, A., Bardy, S., Pernier, S. y Bonsack C. (2011). Improving insight into delusions: A pilot study of metacognitive training for patients with schizophrenia. Journal of Advanced Nursing, 67, 401-407.

Favrod, J., Rexhaj, S., Bardy, S., Ferrari, P., Hayoz, C., Moritz, S., Conus, P. y Bonsack, C. (2013). Sustained antipsychotic effect of metacognitive training in psychosis: A randomized-controlled study. European Psychiatry, 29, 275-281.

Freeman D. (2007). Suspicious minds: The psychology of persecutory delusions. Clinical Psychology Review, 27, 425-457.

Haddock, G., McCarron, J., Tarrier, N. y Faragher, E. B. (1999). Scales to measure dimensions of hallucinations and delusions: The psychotic symptom rating scales (PSYRATS). Psychological Medicine, 29, 879-889.

Kane J. M. (2007). Treatment resistant schizophrenic patients. Journal of Clinical Psychology, $57,35-40$. 
Kay, R., Fiszbein, A. y Opler, A. (1987). The Positive and Negative Syndrome Scale (PANSS) for Schizophrenia. Schizophrenia Bulletin, 13, 261-276.

Kay, R. y Sevy, S. (1990). Pyramidical model of schizophrenia. Schizophrenia Bulletin, 16, 537545.

Kumar, D., ZiaUlHaq, M., Dubey, I., Dotivala, K. N., Siddiqui, S., Prakash, R., Abhishek, P. y Nizamie, S. H. (2010). Effect of metacognitive training in the reduction of positive symptoms in schizophrenia. European Journal of Psychotherapy \& Counselling, 12, 149-158.

Lemos Giráldez, S., Vallina Fernández, O., Fernández Iglesias, P., Fonseca, E. y Paino, M. (2010). Bases clínicas para un nuevo modelo de atención a las psicosis / New trends in treatment for psychosis. Clínica y Salud, 21, 299-318.

López-Ilundain, J. M., Pérez-Nievas, F., Otero, M. y Mata, I. (2006). Inventario de Experiencias Delirantes de Peters (PDI) en población general española: fiabilidad interna, estructura factorial y asociación con variables demográficas. Actas Españolas de Psiquiatría, 34, 94-104.

McLeod, H. J., Gumley, A. L., Macbeth, A., Schwannauer, M. y Lysaker, P. H. (2014). Metacognitive functioning predicts positive and negative symptoms over 12 months in first episode psychosis. Journal of Psychiatry Research, 54, 109-15.

Moritz, S., Andreou, C., Klingberg, S., Thoering, T. y Peters, M. J. (2013). Assessment of subjective cognitive and emotional effects of antipsychotic drugs. Effect by defect? Neuropharmacology, 72, 179-186.

Moritz S., Kerstan A., Veckenstedt R., Randjbar S., Vitzthum F., Schmidt C. y cols. (2011). Further evidence for the efficacy of a metacognitive group training in schizophrenia. Behaviour Research and Therapy, 49, 151-157.

Moritz, S., Veckenstedt, R., Bohn, F., Hottenrott, B., Scheu, F., Randjbar, S. y cols. (2013). Complementary group Metacognitive Training (MCT) reduces delusional ideation schizophrenia. Schizophrenia Research, 151, 61-69.

Moritz S., Veckenstedt R., Randjbar S., Vitzthum F. y Woodward T. S. (2011). Antipsychotic treatment beyond antipsychotics: Metacognitive intervention for schizophrenia patients improves delusional symptoms. Psychological Medicine, 41, 1823-1832.

Moritz, S., Vitzthum, F., Randjbar, S., Veckenstedt, R. y Woodward, T. S. (2010). Detecting and defusing cognitive traps: Metacognitive intervention in schizophrenia. Current Opinion in Psychiatry, 23, 561-569.

Moritz, S., Voight, M., Köther, U., Leighton, L., Kjahili, B., Babur, Z., Jungclaussen, D. y cols. (2014). Can virtual reality reduce reality distortion? Impact of performance feedback on symptom change in schizophrenia patients. Journal of Behavior Therapy \& Experimental Psychiatry, 45, 267-271.

Moritz, S. y Woodward, T. S. (2007). Metacognitive training for schizophrenia patients (MCT): A pilot study on feasibility, treatment adherence, and subjective efficacy. German Journal of Psychiatry, 10, 69-78.

Moritz, S., Woodward, T. S. y Burlon, M. (2005). Metacognitive skill training for patients with schizophrenia (MCT) Manual. Hamburg: Van Ham Campus.

Naughton, M., Nulty, A., Abidin, Z., Davoren, M., O'Dwye,r S. y Kennedy, H. G. (2012). Effects of group metacognitive training (MCT) on mental capacity and functioning in patients with psychosis in a secure forensic psychiatric hospital: A prospective-cohort waiting list controlled study. BMC Research Notes, 5, 302.

Peters, E., Joseph, S., Day, S. y Garety, P. (2004). Measuring Delusional Ideation: The 21-Item Peters y cols. Delusions Inventory (PDI). Schizophrenia Bulletin, 30, 1005-1022. 
Rocha, N. B. y Queirós, C. (2013). Metacognitive and social cognition training (MSCT) in schizophrenia: A preliminary efficacy study. Schizophrenia Research, 150, 64-68.

Woodward, T. S., Moritz, S. y Chen, E. Y. (2006). The contribution of a cognitive bias against disconfirmatory evidence (BADE) to delusions: a study in an Asian sample with first episode of schizophrenia spectrum disorders. Schizophrenia Research, 83, 297-298. 